\title{
Patrones de herbivoría en Vassobia breviflora (Solanaceae): variación en el daño foliar y selección natural mediada por herbívoros
}

\author{
Mariana Valoy ${ }^{1}$, Mariano Ordano ${ }^{1,2}$, Facundo Bernacki ${ }^{1}$, Facundo X. Palacio ${ }^{3}$, \\ Juan Carlos López-Acosta ${ }^{4} \&$ Omar Varela ${ }^{1,5}$ \\ 1. Fundación Miguel Lillo, San Miguel de Tucumán, Tucumán, Argentina; mevaloy@lillo.org.ar, \\ facundobernacki@gmail.com \\ 2. Fundación Miguel Lillo y Unidad Ejecutora Lillo (UEL), Consejo Nacional de Investigaciones científicas y Técnicas \\ (CONICET), San Miguel de Tucumán, Tucumán, Argentina; maordano@lillo.org.ar \\ 3. Facultad de Ciencias Naturales y Museo, Universidad Nacional de La Plata y Consejo Nacional de Investigaciones \\ Científicas y Técnicas (CONICET), Buenos Aires, Argentina; facundo_palacio@fcnym.unlp.edu.ar \\ 4. Centro de Investigaciones Tropicales, Universidad Veracruzana Xalapa, Veracruz, México; carlolopez@uv.mx \\ 5. Instituto de Ambientes de Montañas y Regiones Áridas, Universidad Nacional de Chilecito (UNdeC), Chilecito, La \\ Rioja, Argentina; omarvarela1@gmail.com
}

Recibido 17-I-2018. Corregido 27-V-2018. Aceptado 02-X-2018.

\begin{abstract}
Patterns of herbivory in Vassobia breviflora (Solanaceae): variation in foliar damage and natural selection mediated by herbivorous. Herbivore mediated-selection shapes the evolution of defensive plant traits. Knowledge about the role of herbivores as mediators of selection is scarce and even more if herbivore functional groups are considered. The objectives of this work were (1) to describe the variation in foliar traits between populations and both between and intra-plants within a population, (2) to explore the relationship between the variation in the herbivory level and foliar traits, (3) to determine the relationship between leaf traits and damage patterns and (4) estimate the selection regimes by different herbivore functional groups. We conducted this study in four populations of Vassobia breviflora in Northwestern Argentina (Yungas). The foliar traits considered were size, leaf area (af), shape (leaf length / width ratio; laf) and proportion of leaf area removed (pafr) $(\mathrm{N}=1582$ leaves, 57 plants). The herbivores consumed $15.6 \%$ of the leaf area and $76.8 \%$ of the variation in the pafr occurred at the sub-individual level. The damage pattern was dominated by cutter herbivores $(70 \%)$, followed by a dotted herbivory pattern $(22 \%)$, mixed $5 \%$ and $1 \%$ miner. Nonlinear selection was detected for laf $(\gamma \mathrm{ii}=-0.180 ; \mathrm{EE}=0.76 ; \mathrm{P}<0.05)$, and correlational selection between the cutter damage and $\mathrm{af}(\gamma \mathrm{ij}=-1.297$; $\mathrm{SE}=0.62 ; \mathrm{P}<0.05)$ and between the dotted damage and $\mathrm{af}(\gamma \mathrm{ij}=-1.130 ; \mathrm{SE}=0.76 ; \mathrm{P}<0.05)$. Natural selection favored plants with small leaves and high foliar removal and large leaves with less damage and selection against larger leaves with greater damage was detected. In addition, deduced from the relationship between the damage type and the relative fitness, the selection would favor the dotted damage over the cutter one. The plants would resolve the conflict with the herbivores according to the damage type and natural selection would regulate the foliar display as a strategy to deal with the herbivore diversity.
\end{abstract}

Key words: plant-animal interactions; phenotypic selection; functional groups; leaf traits; herbivory levels; damage patterns.

Valoy, M., Ordano, M., Bernacki, F., Palacio, F. X., López-Acosta, J. C., \& Varela, O. (2018). Patrones de herbivoría en Vassobia breviflora (Solanaceae): variación en el daño foliar y selección natural mediada por herbívoros. Revista de Biología Tropical, 66(4), 1683-1700.

La herbivoría es una interacción entre plantas y animales en donde los últimos consumen tejido vivo de las plantas (hojas, tallos, flores, frutos, raíces); esta interacción, afecta negativamente el desempeño y la adecuación de las plantas (por ejemplo, crecimiento, sobrevivencia, reproducción, habilidad competitiva) (Crawley, 1997). Por consiguiente, los 
herbívoros se consideran entre los principales agentes de selección que dirigen y moldean el fenotipo de las plantas. Este proceso está mediado por la intensidad de daño y en consecuencia tiene el potencial de modular la magnitud de la selección natural sobre el fenotipo (Pilson, 2000; Valverde, Fornoni, \& NúñezFarfán, 2001; Hare, 2012; Ågren, Hellström, Toräng, \& Ehrlén, 2013).

La evidencia empírica señala que el daño por herbívoros es heterogéneo y su condición se asocia a las múltiples fuentes de variación que modulan los niveles de daño y en consecuencia la magnitud de las presiones de selección (Strauss, Rudgers, Lau, \& Irwin, 2002; Cárdenas, Valencia, Kraft, Argoti, \& Dangles, 2014). A pesar de que una extensa bibliografía reconoce el papel selectivo de los herbívoros (Marquis, 1992; Johnson et al., 2012; Marquis et al., 2016), son escasos los trabajos que abordan en conjunto: i) las fuentes de variación del daño; ii) variación en los niveles de herbivoría y; iii) los patrones de daño en función al tipo de herbívoro, desde una perspectiva micro-evolutiva, que permita dilucidar y comprender la forma y dirección con que opera la selección natural mediada por herbívoros sobre rasgos de las plantas.

En general, las restricciones ontogenéticas, filogenéticas, fisiológicas, así como las condiciones ambientales, rigen la variación en características morfológicas y de desarrollo de las plantas (Pigliucci, 2003; Herrera, 2009). Una porción de tal variación es atribuible al resultado de presiones de selección ejercidas por los herbívoros (Gilbert, 1979; Givnish, 1990; Brown, Lawton, \& Grubb, 1991; Campitelli, Simonsen, Wolf, Manson, \& Stinchcombe, 2008). Por lo tanto, la evaluación de la expresión de los rasgos foliares involucrados en la interacción con los herbívoros, así como los patrones de forrajeo entre grupos funcionales de herbívoros, son esenciales para esclarecer las fuentes de variación fenotípica en rasgos de las plantas. La elección de características foliares por herbívoros y el consumo de área foliar varían entre grupos de herbívoros y entre hojas de distinta etapa de desarrollo, lo cual determina los patrones de forrajeo (Mauricio,
Bowers, \& Bazzaz, 1993; Hikosaka, 2005; Shimada, Takahashi, Shibata, \& Yagihashi, 2015) los cuales condicionarán la intensidad y variabilidad del daño a nivel intra-planta, entre plantas o entre poblaciones con consecuencias asociadas en el éxito reproductivo.

Dependiendo de los niveles de organización, podríamos esperar diferentes consecuencias de la variación de atributos de las plantas en función al ataque de los herbívoros. A nivel poblacional, el impacto diferencial de los herbívoros promueve mayor variación en la dirección y magnitud de la selección natural y mantiene la variación de los rasgos de defensa de las plantas (Wade \& Kalisz, 1990; Valverde et al., 2001; Canto Aguilar, 2004; MacColl, 2011). A nivel individual, la variación en el daño resulta de las diferentes características fenotípicas que expresan los individuos de una población (Strauss, Conner, \& Lehtilä, 2001). A nivel sub-individual, la organización modular de las plantas proporciona una gama de ambientes y recursos (hojas, flores, frutos, semillas) de diferente calidad nutritiva que incrementan la variación en los rasgos y limitan o favorecen la respuesta y consecuente adaptación de los herbívoros (Whitham, 1983; Baldwin \& Schultz, 1983; Herrera, 2009). En conjunto, la magnitud de la selección natural mediada por herbívoros dependerá del valor nutricional de los órganos consumidos, de la intensidad y del tipo de daño producido por los herbívoros (Dirzo, 1984; Hendrix, 1988; Mauricio et al., 1993; Lehtilä, 1999; Warner \& Cushman, 2002).

En general, las fuerzas de selección que ejercen los herbívoros sobre las plantas, son difíciles de detectar y por tal razón, no deberían considerarse relevantes en la evolución de rasgos de las plantas (Bernays \& Graham, 1988; Schmitt, Hay, \& Lindquist, 1995). Otro enfoque señala que el impacto selectivo de los herbívoros es altamente variable en el tiempo y el espacio (Coley, Bryant, \& Chapin, 1985; Lehndal \& Ågren, 2015), lo cual resulta en escenarios complejos que serían esencialmente el reflejo de las diferentes presiones selectivas impuestas por los herbívoros y sus diferentes 
formas de expresión sobre la planta (por ejemplo, grupos funcionales como minadores, agalleros, cortadores) (Thompson, 2005; Geber \& Giffen, 2003; Ågren et al., 2013). Ambos enfoques señalan claras rutas de investigación las cuales esclarecerían la relación entre los patrones de daño de los herbívoros y la intensidad de las presiones de selección.

En este trabajo, se describe la variación en los rasgos foliares (tamaño, forma y desarro1lo), y se explora su relación con los patrones de daño y niveles de herbivoría, en cuatro poblaciones de Vassobia breviflora (Solanaceae). Además, se estimaron los regímenes de selección mediada por diferentes grupos funcionales de herbívoros. Específicamente se plantean las siguientes preguntas: (1) ¿Cuáles son los patrones de variación en la herbivoría asociada a los rasgos foliares y como varían a diferentes niveles de organización (entre poblaciones, entre plantas y dentro de plantas)? (2) ¿Las características foliares condicionan los patrones de daño y en consecuencia determinan los niveles de herbivoría? (3) ¿Distintos grupos funcionales de herbívoros ejercen diferentes presiones selectivas sobre los rasgos de las plantas?

\section{MATERIALES Y MÉTODOS}

Área de estudio: El trabajo se realizó en cuatro sitios del noroeste de Argentina. El área corresponde a la Provincia Fitogeográfica de las Yungas (Cabrera, 1976), la cual constituye el extremo meridional de una región natural denominada Selva Tucumano-Boliviana (Hueck, 1978). Las Yungas se desarrollan en las laderas húmedas de las montañas, a lo largo de 1200 km, desde Santa Cruz (Bolivia) hasta Catamarca (Argentina). Las precipitaciones son marcadamente estacionales y se concentran entre noviembre y marzo (Brown, Grau, Lomáscolo, \& Gasparri, 2002). Las condiciones ambientales donde se desarrollan las cuatro poblaciones son similares a nivel regional y pertenecen a la misma zonación fitogreográfica; las cuales se describen a continuación:
1. San Ignacio $\left(23^{\circ} 52^{\prime} 33.86^{\prime \prime} \mathrm{S}-63^{\circ} 22^{\prime}\right.$ 17.95" W, 550 m.s.n.m.; Departamento Orán, provincia de Salta). Es el sitio más septentrional y se ubica en el sector inferior de las Yungas de la Alta Cuenca del Río Bermejo. Las especies dominantes son de las familias Myrtaceae, Lauraceae y Solanaceae. Las precipitaciones varían de 1000 a $1400 \mathrm{~mm}$ anuales (Bianchi \& Yáñez, 1992).

2. Villa Padre Monti $\left(26^{\circ} 30^{\prime} 36.94 " \mathrm{~S}-64^{\circ}\right.$ 59'38.07" W, 950 m.s.n.m.; Departamento Burruyacu, provincia de Tucumán; a partir de aquí referido como V. P. Monti). Está representado por bosques que se encuentran fragmentados por áreas de cultivos y las especies dominantes pertenecen a las familias Fabaceae y Juglandaceae. Las precipitaciones varían de 700 a $800 \mathrm{~mm}$ anuales (Santillán de Andrés, Barbieri de Santamarina, Ricci, \& Würschmidt, 1967).

3. Reserva Provincial Aguas Chiquitas $\left(26^{\circ} 35^{\prime} 53.82\right.$ " S - 65'10'44.95" W, 700 m.s.n.m.; Departamento Tafí Viejo, provincia de Tucumán; a partir de aquí referido como A. Chiquitas). Se caracteriza por presentar sectores de Selva Montana con elementos de transición y la vegetación se compone principalmente por árboles perennifolios (Myrtaceae y Myrsinaceae). Las precipitaciones varían de 800 a $900 \mathrm{~mm}$ anuales (Santillán de Andrés et al., 1967).

4. Toma del Río Horqueta (Reserva Provincial La Florida) $\left(27^{\circ} 07^{\prime} 21.64 " \mathrm{~S}\right.$ - 6546'48.57’ W, 1100 m.s.n.m.; Departamento Monteros, provincia de Tucumán; a partir de aquí referido como La Horqueta). Se encuentra enclavado en las laderas orientales de la Sierra del Aconquija. La vegetación corresponde a la Selva Montana y las especies dominantes pertenecen a las familias Lauraceae y Sapindaceae. Las precipitaciones varían entre 1600 y $1800 \mathrm{~mm}$ anuales (Ayarde, 2005; Brown \& Corcuera, 1999). 
Especie de estudio: Vassobia breviflora (Sendtn.) Hunz. (Solanaceae), es un arbusto o pequeño árbol nativo perennifolio, con registros máximos de $15 \mathrm{~m}$ de altura y se ramifica desde la base. Se distribuye en el noroeste y centro de Argentina, Bolivia, Paraguay, Uruguay y Brasil (Cabrera, 1979; Anton \& Zuloaga, 2013). Las hojas son simples, alternas, lámina elíptica de $4-12 \mathrm{~cm}$ longitud y $2-4 \mathrm{~cm}$ de ancho, pecíolo 1-2 cm longitud con una longevidad promedio de 6 meses (Valoy, sin publicar). Puede presentar espinas en tallos y ramas, las flores y los frutos se disponen en fascículos sobre braquiblastos cortos (Bernacki, 2014, Bernacki, Albornoz, Valoy, \& Ordano, 2015). Las hojas son consumidas por insectos pertenecientes a los órdenes Lepidóptera y Coleóptera (Valoy, obs. per.). Las flores son polinizadas por insectos, principalmente himenópteros de la familia Apidae y Colletidae (Taura \& Laroca, 2004, Bernacki, 2014) y los frutos son dispersados principalmente por aves (Giannini, 1999; Palacio et al., 2017). Florece en primavera y fructifica en verano (Digilio \& Legname, 1966). Crece en bordes de bosques desde el nivel del mar hasta los 2000 m.s.n.m., con requerimiento intermedio de luz y es pionera en la sucesión vegetal (Anton \& Zuloaga, 2013).

\section{Recolecta de campo y medición de varia-}

bles: Teniendo en cuenta las cuatro poblaciones, se seleccionaron en total 56 individuos adultos y en estado reproductivo de $V$. breviflora. La selección de plantas se hizo según el acceso a las plantas, dado que es una especie que crece en bordes de camino y en zonas peridomésticas (Anton \& Zuloaga, 2013). En cada individuo se recolectaron entre 15 y nueve hojas adultas (de la base de ramas) y jóvenes (de los extremos de ramas), según la disponibilidad de hojas y los criterios para identificar hojas jóvenes de adultas se basaron en características de apariencia en color y textura de las hojas en campo. En total se obtuvieron 1582 hojas, de las cuales 988 correspondieron a la población de Aguas Chiquitas $(\mathrm{N}=35$ plantas), 287 a V. P. Monti $(\mathrm{N}=10), 163$ a San Ignacio $(\mathrm{N}=6)$ y $144 \mathrm{a} \mathrm{La}$ Horqueta $(\mathrm{N}=5)$.
La recolecta de hojas se realizó en un rango 1 a $3 \mathrm{~m}$ sobre el nivel del suelo. Las hojas fueron fotografiadas (cámara digital Nixon Coolpix P510) y procesadas con el programa ImageJ versión 1.4g (Rasband, 1997). Para cada hoja (dañada o no) se obtuvo el área del limbo (área foliar sin pecíolo) $\left(\mathrm{cm}^{2}\right)$, en adelante "área observada". En las hojas dañadas, la forma de la hoja original (previa al daño) fue inferida siguiendo la forma del área foliar restante. El área foliar removida (afr) fue la diferencia entre área foliar estimada y el área foliar observada. La proporción de área foliar removida (pafr) se obtuvo mediante la fórmula pafr $=$ afr / área foliar (observada o estimada). Este estimador es una medida fácil y precisa de cuantificar la medida del daño puntual en la hoja (O'Neill, Aitken, King, \& Alfaro, 2002; Valoy, Ordano, \& Benavídez, 2012). Para las cuatro poblaciones, la herbivoría foliar fue exclusiva de insectos y se caracterizaron los diferentes grupos funcionales de herbívoros en relación a los tipos de daño. Se discriminó la pafr correspondiente a cada grupo funcional, los cuales fueron definidos según el tipo de daño foliar: "cortador", "punteador" y "minador". Los primeros refirieron a la remoción de lámina foliar incluyendo el borde de las hojas, caracterizado por el típico daño por larvas de lepidópteros y ortópteros. Los punteadores se definieron por daños dentro de la lámina foliar usualmente pequeños, múltiples y asociados a coleópteros, e himenópteros. El daño "minador" se visualizó como galerías (o minas) en las hojas. Las minas son de tamaño y forma variable y son la huella que dejan las larvas de lepidópteros, dípteros o himenópteros al alimentarse del mesófilo foliar específicamente en el tejido esponjoso y de empalizada. El daño combinado (o mixto) se definió como la presencia de más de un tipo de daño en la lámina foliar. Los daños que no pudieron atribuirse a los herbívoros (por ejemplo daño físico) se consideraron en la categoría "indeterminado".

El ancho y longitud de la hoja se utilizaron para el cálculo del cociente longitud / ancho (laf). El área foliar (af) $\left(\mathrm{cm}^{2}\right)$ y laf constituyen descriptores de características foliares (tamaño 
y forma) que han sido señalados como potenciales rasgos blanco de selección natural mediada por herbívoros (Campitelli et al., 2008).

Como variable de éxito reproductivo se utilizó el tamaño de la cosecha el cual se definió como el número de frutos maduros por planta. Se contó el número total de fascículos por planta y el número de ramas principales. Diez fascículos fueron seleccionados al azar en una rama principal y se contó el número de frutos maduros en cada fascículo. El tamaño de la cosecha fue estimado como: $\mathrm{C}=\mathrm{F}$ x I x $\mathrm{R}$, donde $\mathrm{C}$ es el tamaño de la cosecha, $\mathrm{F}$ es el promedio de frutos maduros por fascículos en una rama principal, I es el número de fascículos en una rama principal y $\mathrm{R}$ es el número de ramas principales.

Para describir las plantas y los rasgos foliares (afr, pafr, af y laf) en cada población, se calcularon las medidas media aritmética (x), el desvío estandar (de) y el coeficiente de variación (cv).

Para explorar el efecto de la edad de la hoja (o desarrollo) y el tipo de daño sobre la proporción de área foliar removida (pafr), el tamaño (af) y la forma de la hoja (laf), se aplicaron modelos lineales generales mixtos (Zuur, Ieno, Walker, Saveliev, \& Smith, 2009). Estos modelos tienen en cuenta la estructura agregada de los datos dentro de distintos niveles que pueden (o no) estar ordenados de manera jerárquica y permiten combinar efectos fijos y aleatorios dentro de un modelo. Los efectos fijos son los efectos de las variables predictoras sobre la media de la variable respuesta, mientras que los aleatorios, son los efectos de la variación entre y dentro de los niveles de un factor (entre sitios, entre plantas y dentro de la planta) sobre la variable respuesta. Además, los modelos mixtos admiten datos con estructura desbalanceada (Gelman \& Hill, 2007) lo cual es compatible con nuestro modelo ya que el tamaño de muestra fue diferente en cada población. Se construyeron tres modelos (uno para cada variable de respuesta: af, laf, pafr), en todos los casos las variables edad de la hoja y tipo de daño se incluyeron como efectos fijos con sus interacciones, mientras que sitio y planta se incluyeron como factores aleatorios. Para explorar el efecto de la fracción (afr) y proporción de área foliar removida (pafr) sobre el tamaño y forma de la hoja, la variable afr se adicionó al modelo que contenía af como variable de respuesta y la variable pafr se incluyó en el otro modelo que contenía laf como variable de respuesta y en cada modelo ambas variables fueron incluidas como efectos fijos. Para evitar problemas de colinealidad entre las variables, pafr no fue incluida como variable explicatoria en el primer modelo (modelo con af como variable respuesta) en su lugar, se incluyó afr ya que ésta representa el área foliar removida sin corrección para el tamaño de la hoja (ver calculo de pafr en medición de variables). A partir de estos modelos, se estimaron los componentes de varianza que permiten discernir la magnitud de la variación entre sitios, entre plantas y dentro de la planta, incluyendo el tipo de daño, y brindan información de la proporción de varianza que puede ser atribuida a cada nivel del factor.

Los porcentajes de variación atribuidos a cada nivel se calcularon como el cociente: componente de varianza/suma de los componentes de varianza (Crawley, 2007; Herrera, 2009) (fórmula en $\mathrm{R}=$ variable respuesta $\sim$ variable explicatoria, random $=\sim 1 \mid$ sitio/planta). Los niveles del factor tipo de herbivoría fueron reducidos (a dos categorías) y se conservaron cortador y punteado. En consecuencia, el sitio San Ignacio fue excluido del análisis ya que no presentó estos tipos de daño.

Adicionalmente, se evaluó el efecto de las variables explicatorias (tipo de herbivoría y edad de la hoja) sobre la pafr (variable de respuesta). En este caso, se ajustó un modelo de regresión beta (Ferrari \& Cribari-Ñeto, 2004). El análisis final incluyó las dos variables explicatorias. Para examinar la relación entre los sitios y la pafr, se agregó sitio como variable explicatoria y la interacción entre sitio y tipo de herbivoría.

Para evaluar las características foliares que podrían ser blanco de selección y examinar la selección ejercida por cada grupo funcional de herbívoro, se aplicó un análisis de selección 
natural fenotípica (Lande \& Arnold, 1983). La selección natural se mide como una función entre el fenotipo y la adecuación (éxito reproductivo). Como medida de éxito reproductivo se consideró el número de frutos por planta (tamaño de la cosecha) y los rasgos fueron la proporción de área foliar removida discriminada por tipo de daño: insectos cortadores (cortador), punteadores (punteado), área foliar (af) y la relación longitud/ancho (laf). Si bien, el pafr como rasgo blanco de la selección natural no constituye un rasgo fenotipico por si mismo, incluirlo en estudios de selección es muy valioso, dado que representan rasgos complejos y pueden considerarse componentes extendidos del fenotipo (Weis \& Gorman, 1990; Weis, Abrahamson, \& Andersen, 1992; Gerasymenko, 2017).

La distribución no normal de los errores del modelo se resolvió con la aplicación de Modelos Lineales Generalizados (GLM, Zuur et al., 2009) con una distribución del error de tipo Poisson corregido por sobredispersión. Como primer paso se estandarizaron las variables sin transformar y los valores de los estimadores (gradientes en este caso) se tomaron del modelo con la adecuación relativa (Wrel) como variable de respuesta (sensu Lande \& Arnold, 1983), mientras que la significancia se obtuvo a partir del modelo lineal generalizado con la adecuación sin relativizar. Todos los análisis fueron realizados con R 3.2.1 (R Development Core Team, 2013) y se utilizaron los paquetes nlme (Pinheiro, Bates, DebRoy, \& Sarkar, 2012), betareg (Simas \& Rocha, 2015), lattice (Sarkar, 2015) y visreg (Breheny \& Burchett, 2015). Las bases de datos y las rutinas correspondientes se presentan en el Apéndice 1.

\section{RESULTADOS}

\section{Estadística descriptiva para las varia-}

bles: Considerando a las cuatro poblaciones, el área foliar removida para $V$. breviflora fue de $15.6 \%$ (Fig. 1). En cuanto a los rasgos por sitio, la población de V. P. Monti exhibió mayor pafr, mientras que A. Chiquitas presentó hojas con mayor af $(20.87$; SD $=11.41)$ y menor laf $(1.91 ; \mathrm{SD}=0.48)$, esto es, hojas grandes y levemente más largas que anchas (Fig. 1). La variable con mayor rango de variación fue af. El cv mas alto correspondió a la variable afr $(\mathrm{cv}=1.42)$ en tres de las cuatro poblaciones, excepto en La Horqueta donde el mayor cv correspondió a la pafr (Apéndice 1).

Tipos de daño y edad de la hoja: En las cuatro poblaciones el tipo de daño más frecuente fue el daño cortador ( $\mathrm{pafr}=70 \%$ ), seguido del daño punteado (51.35\%) y minador (35.95 \%) (Fig. 2). Cuando se discriminó la pafr por población y por tipo de daño, se observó que en La Horqueta $22.4 \%$ de la pafr fue consumida por cortadores, seguido de V. P. Monti, donde los cortadores removieron $21.2 \%$. Al discriminar por edad de la hoja, $24.5 \%$ de las hojas viejas evidenciaron daño por cortadores y $29 \%$ de las hojas nuevas presentaron daño punteado. Cuando se exploró af, discriminando por población y tipo de daño, el valor más alto fue en V. P. Monti. El daño que predominó fue el punteado $(39.15 ; \mathrm{SD}=8.09)$ y prevaleció en las hojas viejas $\left(39.15 \mathrm{~cm}^{2}\right.$; $\left.\mathrm{SD}=8.12\right)$. En cuanto a laf, el valor más alto fue en V. P. Monti para daño cortador $(2.70 ; \mathrm{SD}=2.04)$ y fué mas evidente en las hojas nuevas (2.89; $\mathrm{SD}=1.30)$ (Fig. 2).

Componentes de varianza y relación entre variables: El $84 \%$ de la variación en el rasgo laf fue explicada por la variación dentro de la planta, seguido de $76.84 \%$ en la pafr y $57.74 \%$ en af. Los porcentajes de variación con respecto a los otros niveles (entre plantas y dentro de la planta), mostraron que para el rasgo af, $17.69 \%$ de la variación estuvo explicada entre plantas y el $24.56 \%$ entre sitios.

El af se incrementó con la edad (4.09; EE $=0.78 ; \mathrm{P}<0.01)$ y se relacionó positivamente con el afr $(1.97 ; \mathrm{EE}=0.24 ; \mathrm{P}<0.01)$, probablemente como resultado de mayor tiempo y área de exposición a los herbívoros (Cuadro 1, ver pie de cuadro A). Por otra parte, las hojas con mayor laf presentaron mayor pafr $(1.72 ; \mathrm{EE}=$ $0.50 ; \mathrm{P}<0.01$ ) (Cuadro 1, ver pie de cuadro B). La pafr también se relacionó positivamente con 

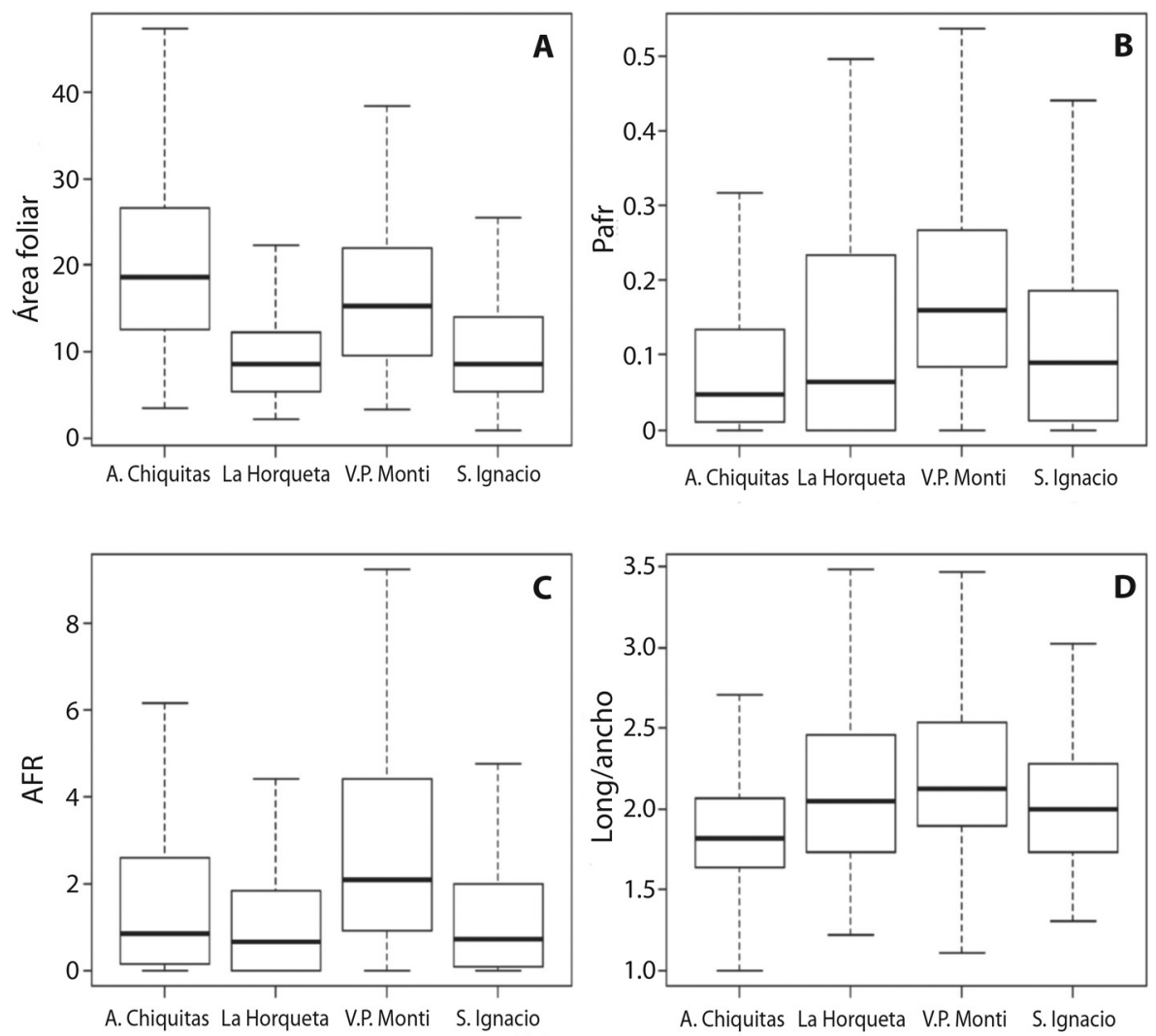

Fig. 1. Variación en rasgos foliares por sitio (La Horqueta, A. Chiquitas, V. P. Monti, S. Ignacio), área foliar; proporción de área foliar removida por insectos herbívoros (Pafr); área foliar removida por insectos herbívoros (AFR) y relación longitud/ ancho del limbo de la hoja (Long/ancho).

Fig. 1. Variation in leaf traits per site (La Horqueta, A. Chiquitas, V. P. Monti, S. Ignacio), leaf area, proportion of leaf area removed by herbivorous insects (Pafr), leaf area removed by herbivorous insects (AFR) and length / width ratio of leaf blade (Length/Width).

la edad de la hoja, siendo mayor en las hojas viejas $(0.23 ; \mathrm{EE}=0.04 ; \mathrm{P}<0.01)$. Respecto a los sitios, se registró mayor pafr en La Horqueta $($ coef $=0.69 ; \mathrm{EE}=0.10 ; \mathrm{P}<0.01)$ y en $\mathrm{V}$. $\mathrm{P}$. Monti $(0.63 ; \mathrm{EE}=0.06 ; \mathrm{P}<0.01)($ Cuadro 2$)$. En lo que respecta al tipo de daño, se encontró una relación negativa entre daño punteado y pafr, sugiriendo que a mayor dominancia del daño punteado, menor es la proporción de área foliar removida de tipo cortador y minador $(-0.26$; $\mathrm{EE}=0.07$; $\mathrm{P}<0.01)$ (Cuadro 2). Asimismo, ninguna de las interacciones entre tipo de daño y sitio fueron significativas.
Selección natural mediada por herbívoros: encontramos selección cuadrática y correlacional para los rasgos foliares y el tipo de daño en la población de A. Chiquitas (Cuadro 3). El gradiente cuadrático para el rasgo que describe la forma de la hoja (laf) fue negativo y significativo ( $\gamma \mathrm{ii}=-0.180 ; \mathrm{EE}=0.76 ; \mathrm{P}<$ 0.05) (Fig. 3), lo que indica una relación no lineal entre la forma de la hoja y la adecuación y sugiere que la selección natural favorecería plantas con hojas no muy largas y levemente anchas. Se encontró selección correlacional negativa entre el daño cortador y el área foliar 

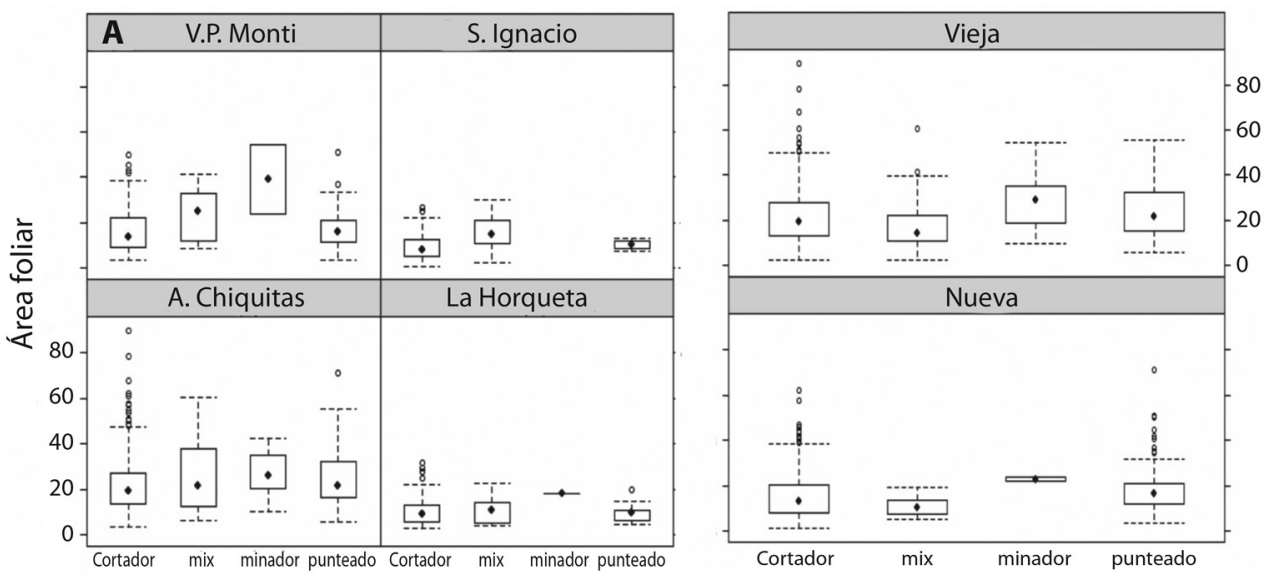

Tipo de herbivoria
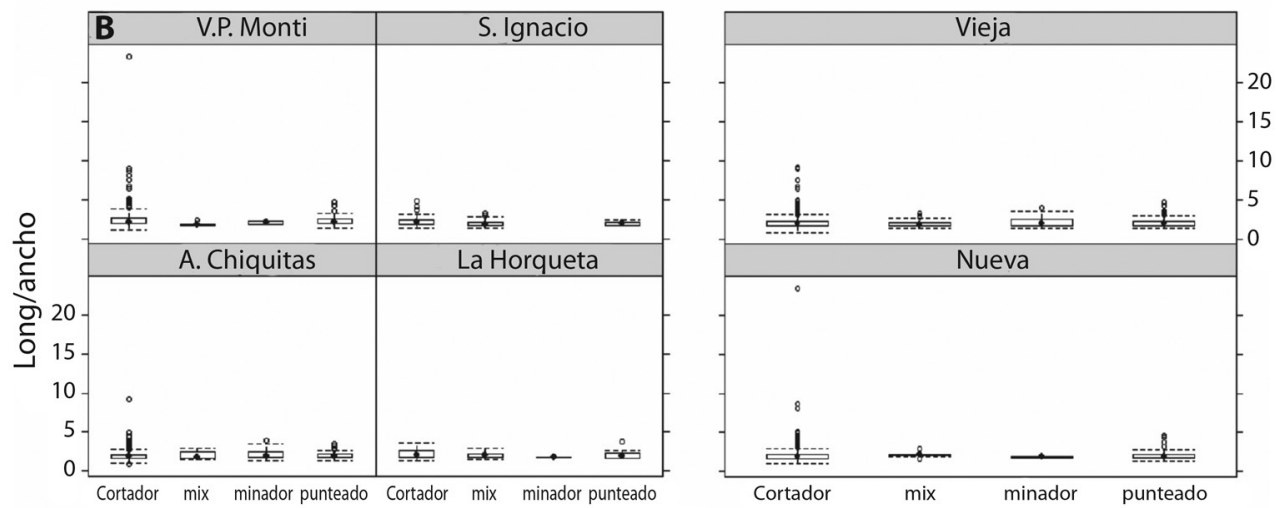

Tipo de herbivoria
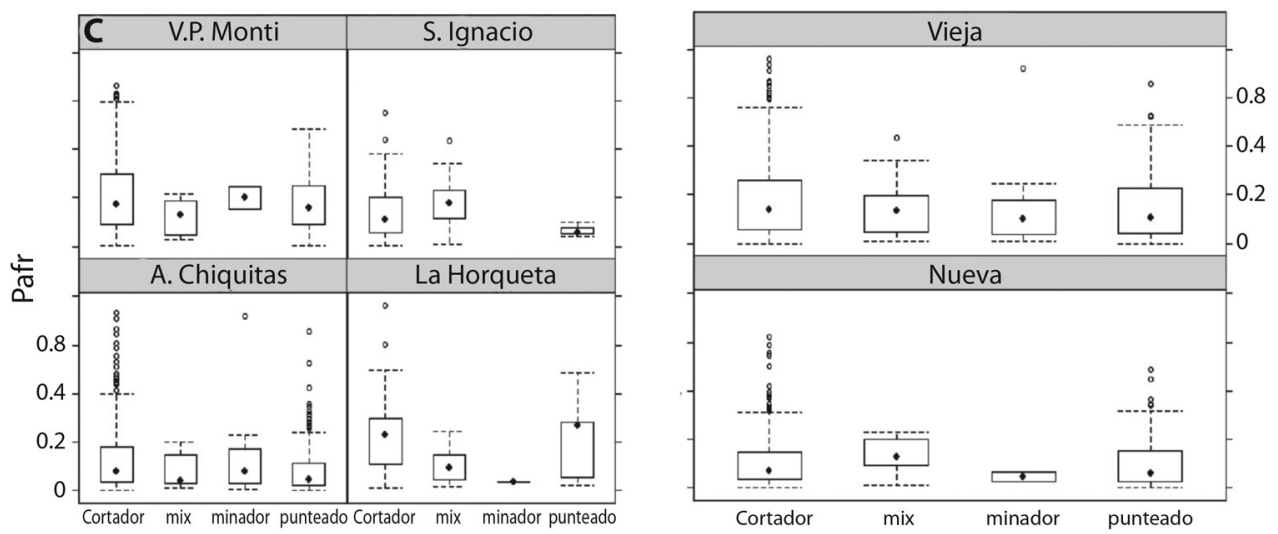

Tipo de herbivoria

Fig. 2. Variación en área foliar (A); relación longitud/ancho de la hoja (B) y proporción de área foliar removida (C), por sitio, tipo de herbivoría y edad de la hoja.

Fig. 2. Variation in leaf area (A); leaf length / leaf width ratio (B) and proportion of leaf area removed (C) by site, herbivory type and leaf age. 
CUADRO 1 / TABLE 1

Modelos lineales generales mixtos / Mixed General Linear Models

\begin{tabular}{lcccc}
\multicolumn{1}{r}{ A } & \multicolumn{2}{c}{ Af } & P \\
Parámetro & Estimador & EE & Z & 0.0023 \\
Intercepto & 9.920 & 3.251 & 3.050 & 0.087 \\
TD & 2.036 & 1.188 & 1.713 & $<0.0001$ \\
EH & 4.097 & 0.787 & 5.206 & $<0.0001$ \\
Afr & 1.976 & 0.248 & 7.952 & 0.786 \\
TDxEH & 0.431 & 1.589 & 0.271 & 0.994 \\
AFRxTD & 0.003 & 0.479 & 0.007 & 0.0024 \\
AFRxEH & -0.800 & 0.263 & -3.043 & 0.628 \\
AFRxEHxTD & -0.248 & 0.513 & -0.483 & $\mathrm{P}$ \\
& & & & $<0.0001$ \\
Parámetro & Estimador & LE & Z & 0.705 \\
Intercepto & 1.997 & 0.183 & 10.872 & 0.847 \\
TD & 0.051 & 0.137 & 0.378 & 0.893 \\
EH & -0.018 & 0.097 & -0.192 & $<0.001$ \\
TDxEH & -0.025 & 0.191 & -0.133 & 0.061 \\
Pafr & 1.727 & 0.508 & 3.397 & 0.471 \\
pafrxTD & -1.776 & 0.947 & -1.873 & 0.285 \\
pafrxEH & -0.414 & 0.575 & -0.720 & 1.069 \\
pafrxEHxTD & 1.220 & 1.140 & & \\
\hline
\end{tabular}

$\mathbf{A}=$ Efecto de tipo de daño (TD), edad de la hoja (EH) y área foliar removida (afr) en función del tamaño de la hoja (Af) en $V$. breviflora.

$\mathbf{B}=$ Efecto de tipo de daño (TD), edad de la hoja (EH) y la proporción de área foliar removida (pafr) en función de la forma de la hoja (Laf) en $V$. breviflora.

$\mathbf{A}=$ Efect of damage type (TD), leaf age (EH) and leaf area removed (afr) as a function of leaf size (Af) in $V$. breviflora .

$\mathbf{B}=$ Efect of damage type (TD), leaf age (EH) and proportion of leaf area removed (pafr) as a function of leaf size (Laf) in $V$. breviflora.
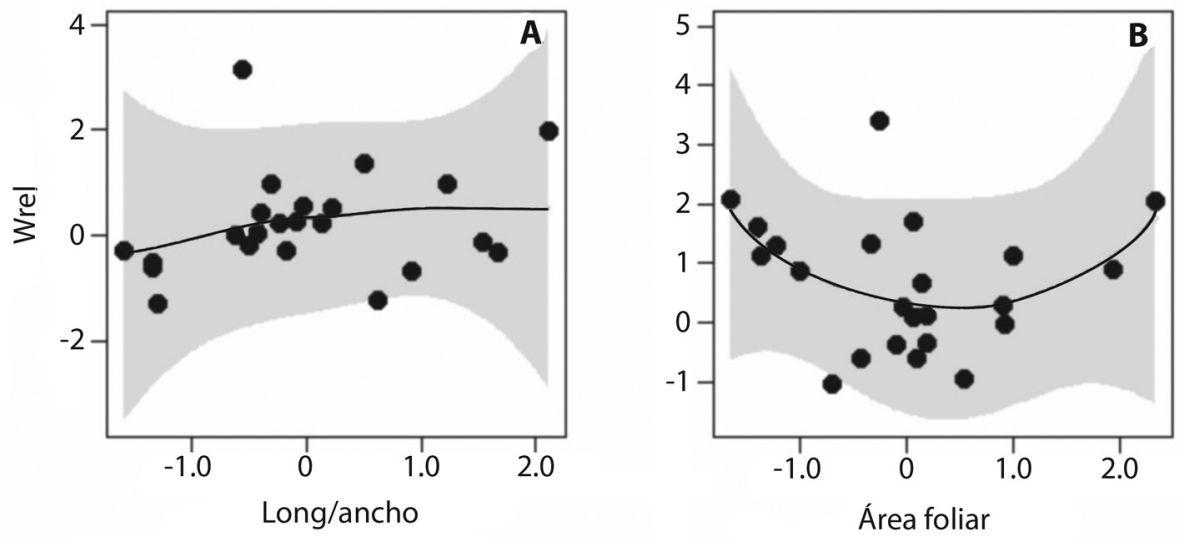

Fig. 3. Selección fenotípica multivariada sobre rasgos foliares. Gradientes de selección no lineal (A. relación longitud ancho/ ancho de la hoja y B. área foliar) en una población de $V$. breviflora en Aguas Chiquitas ( $\mathrm{N}=23$ plantas). Wrel representa el número de frutos por planta relativizado a la media poblacional

Fig. 3. Multivariate phenotypic selection on leaf traits. Non-linear selection gradients in a $V$. breviflora population, Aguas Chiquitas ( $\mathrm{N}=23$ plants). Wrel represents the number of fruits per plant relativized to its population mean. 
CUADRO 2 / TABLE 2

Regresión Beta / Summary Beta Regression

\begin{tabular}{lcccc}
\multicolumn{1}{r}{ Parámetro } & \multicolumn{2}{c}{ Pafr } & P \\
Intercepto & Estimador & SE & z & $<0.0001$ \\
EH:HV & -2.215 & 0.049 & -44.330 & $<0.0001$ \\
S:LH & 0.230 & 0.049 & 8.765 & $<0.0001$ \\
S:VPM & 0.698 & 0.105 & 6.595 & $<0.0001$ \\
S:SI & 0.632 & 0.068 & 9.226 & 0.015 \\
TD:M & 0.230 & 0.095 & 2.416 & 0.235 \\
TD:P & -0.201 & 0.169 & -1.187 & $<0.0001$ \\
S:LH*TD:M & -0.266 & 0.077 & -3.440 & 0.016 \\
S:VPM*TD:M & -0.640 & 0.266 & -2.406 & 0.383 \\
S:SI*TD:M & -0.292 & 0.336 & -0.872 & 0.266 \\
S:VPM*TD:P & 0.259 & 0.233 & 1.111 & 0.104 \\
S:SI*TD:P & 0.203 & 0.125 & 1.626 & 0.727
\end{tabular}

$\mathbf{H V}=$ hoja vieja, $\mathbf{V P M}=$ Villa Padre Monti, $\mathbf{S I}=$ San Ignacio, $\mathbf{L H}=$ La Horqueta, $\mathbf{M}=$ daño minador, $\mathbf{P}=$ daño punteado. Efecto de edad de la hoja (EH), sitio (S) y tipo de daño (TD) sobre la proporción de área foliar removida (Pafr) en $V$. breviflora.

$\mathbf{H V}=$ old leaf, $\mathbf{V P M}=$ Villa Padre Monti, $\mathbf{S I}=$ San Ignacio, $\mathbf{L H}=$ La Horqueta, $\mathbf{M}=$ damage minador, $\mathbf{P}=$ dotted damaged Efect of leaf age (EH), site (S) damage type (TD) on the proportion of leaf area removed (Pafr) in V. breviflora.

CUADRO 3 / TABLE 3

Análisis de Selección Fenotípica Multivariada / Multivariate Analysis of Phenotypic Selection

\begin{tabular}{lcc}
\multicolumn{1}{c}{ Rasgo } & $\beta_{\mathrm{i}}(\mathrm{SE})$ & $\gamma_{\mathrm{ii}} \gamma_{\mathrm{ij}}(\mathrm{SE})$ \\
cortador & $-0.241(0.37)$ & $-0.857(0.83)$ \\
punteado & $-0.582(0.36)$ & $1.868(0.93)$ \\
af & $0.127(0.37)$ & $0.812(0.69)$ \\
laf & $-0.371(0.36)$ & $-0.180(0.76)^{*}$ \\
cortador*punteado & & $-0.758(0.75)$ \\
cortador*af & & $-1.297(0.62)^{*}$ \\
cortador*laf & & $0.963(0.57)$ \\
punteado*af & & $-1.138(0.76)^{*}$ \\
punteado*laf & & $0.424(0.49)$ \\
\hline
\end{tabular}

Rasgo: cortador $=$ proporción de área foliar removida por cortadores, punteado $=$ proporción de área foliar removida por punteadores. Wrel $=\mathrm{N}$ de frutos (tamaño de la cosecha), $\boldsymbol{\beta} \mathbf{i}=$ gradientes estandarizados de selección lineal, $\gamma \mathbf{i i}=$ gradientes estandarizados de selección cuadrática, $\gamma \mathbf{i j}=$ gradientes estandarizados de selección correlativa, $\mathbf{S E}=$ errores estándar correspondientes al valor de cada rasgo (valores significativos se indican con *). $\mathrm{N}=23$ de $V$. breviflora, población de Aguas Chiquitas.

Trait: cutter $=$ proportion of leaf area removed by cutters, dotted $=$ proportion of leaf area removed by dotter. Wrel $=$ $\mathrm{N}$ of fruits (crop size), $\mathbf{B i}=$ standardized linear selection gradients, $\gamma \mathbf{i i}=$ standardized quadratic selection gradients, $\gamma \mathbf{i j}=$ standardized correlational selection gradients, $\mathbf{S E}=$ standard error corresponding to the value of each trait (significant values are indicated whith *). $\mathrm{N}=23$ of $V$. breviflora, population of Aguas Chiquitas. 


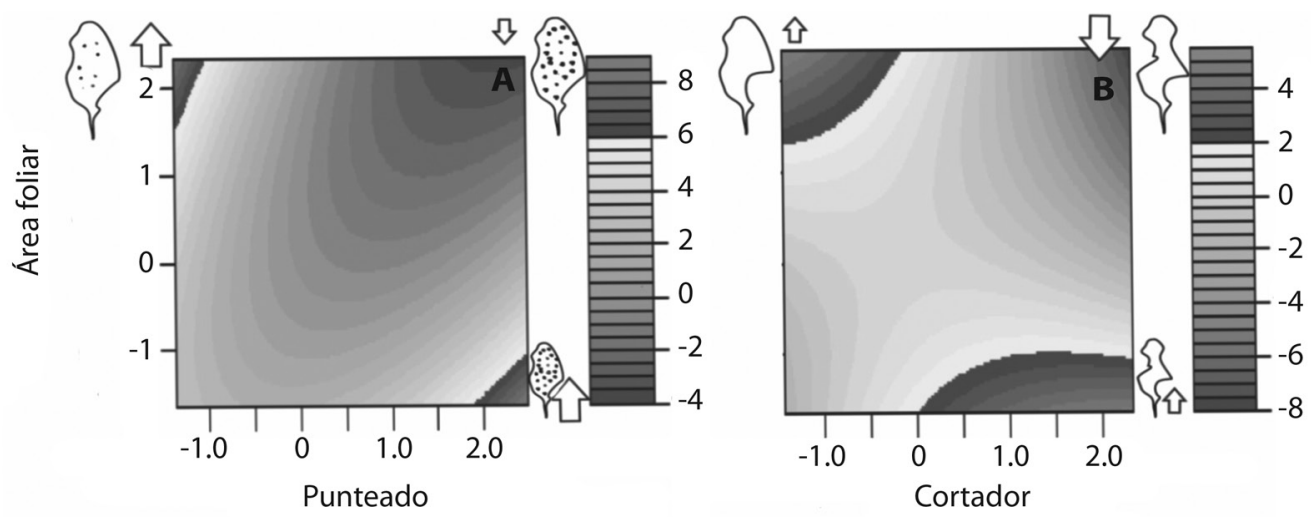

Fig. 4. Selección fenotípica multivariada sobre rasgos foliares (área foliar) y los tipos de daño. Gradientes de selección correlativa (A. daño cortador y B. daño punteado) en una población de $V$. breviflora en Aguas Chiquitas ( $\mathrm{N}=23$ plantas). Superficie de respuesta que muestra la variación en la adecuación a diferentes combinaciones de valores de daño y área foliar. La barra de la derecha muestra la medida de la adecuación (Wrel) representada como el número de frutos por planta relativizado a la media poblacional. El gráfico muestra que plantas con hojas chicas y muy dañadas (extremo inferior derecho) y plantas con hojas grandes y con poco daño (extremo superior izquierdo) incrementan su adecuación, mientras que las plantas con hojas grandes y muy dañadas disminuyen su adecuación (extremo superior derecho). El tamaño de las flechas indica la magnitud de cambio en la adecuación de las plantas (Wrel) para cada situación.

Fig. 4. Multivariate phenotypic selection on leaf traits (leaf area) and damage type. Correlational selection gradients (A. cutter damage and B. dotted damage) in a V.breviflora population, Aguas Chiquitas ( $\mathrm{N}=23$ plants). Response surface showing variation in fitness for different values of damage and leaf area combinations. The bar on the right shows the fitness measure (Wrel) represented as the number of fruits per plant relativized to its population mean. The plot shows that plants with small and very damaged leaves (lower right corner) and plants with large and little damage leaves (upper left) increase their fitness, whereas plants with large and highly damaged leaves decrease their fitness (upper right end). The size of the arrows indicates the magnitude of change in fitness of the plants (Wrel) for each situation.

(af) $(\gamma \mathrm{ij}=-1.297 ; \mathrm{EE}=0.62 ; \mathrm{P}<0.05)$ (Fig. $4 \mathrm{~A})$, y entre el daño punteado y el área foliar $(\gamma \mathrm{ij}=-1.130 ; \mathrm{EE}=0.76 ; \mathrm{P}<0.05)$ (Fig. 4B). Esto es, la selección natural favoreció plantas con hojas de menor tamaño y alta remoción foliar, mientras que hubo selección en contra de las hojas grandes y con abundante daño de tipo cortador y punteado. Esto sugiere que producir hojas de mayor tamaño en un escenario con alto riesgo de herbivoría, además es costoso e independiente del tipo de daño. Sin embargo, a bajos niveles de daño las plantas con hojas grandes mostraron mayor adecuación, lo que sugiere que la selección natural favorecerá un incremento en el despliegue foliar a menor intensidad de daño. Con respecto a la relación entre el tipo de daño y la adecuación, la selección favorecería el daño punteado por sobre el cortador. Las plantas resolverían el conflicto con los herbívoros dependiendo del tipo de daño y la selección natural regularía el despliegue foliar como una estrategia para lidiar con la diversidad de grupos funcionales de herbívoros. Aunque los estimadores en ambos casos fueron de similar magnitud e igual signo, la magnitud del efecto negativo sobre la adecuación fue mayor para la interacción del daño cortador y el área foliar que para el punteado y el área foliar (Fig. 4).

\section{DISCUSIÓN}

Este trabajo desarrolló la hipótesis de que el daño producido por diferentes grupos funcionales de herbívoros y las características foliares morfológicas dirigirían la forma y la dirección de la selección natural. En este sentido, nuestros resultados apoyan la premisa de que la variación en el tamaño y forma de la hoja tiene implicaciones en la adecuación de las plantas, y que se vinculan a características esencialmente reguladoras de la fisiología de 
las plantas (Givnish, 1990). Además, serían el resultado de diferentes presiones selectivas que habrían moldeado y orientado estos rasgos, a fin de minimizar los costos de portar hojas y maximizar la ganancia de carbono en la planta (Lev-Yadun, 2016; Chen et al., 2017).

La selección correlacional para la combinación entre tipo de daño y área foliar mostró similares regímenes selectivos para el daño cortador y para el daño punteado. Linhart (1996) sugiere que los individuos pertenecientes al mismo grupo funcional, y que utilizan un mismo recurso, compartirían similares percepciones sobre los rasgos foliares. Lo inverso sucedería para individuos de grupos funcionales diferentes, dado que la percepción y en consecuencia las presiones selectivas, estarían condicionadas al comportamiento de forrajeo del grupo funcional (Linhart, 1996). No obstante, los regímenes de selección encontrados sugieren que las plantas regularían el costoso despliegue foliar en función al riesgo de daño y no según el tipo de daño.

El efecto diferencial entre los tipos de daño se visualizó por los valores de adecuación para cada patrón de daño. El daño punteado mostró mayor adecuación relativa que el daño cortador. Esto sugiere que las presiones de selección de los grupos funcionales serían distintas y los efectos no se reflejarían en la dirección y forma de la selección, sino en los valores de la adecuación. Para corroborar esta premisa se requeriría conocer cómo operan los mecanismos de asignación de recursos en respuesta a presiones selectivas opuestas (Nguyen, Rieu, Mariani, \& van Dam, 2016). En este sentido, el daño punteado implicaría mayor riesgo que el daño cortador, aunque a nivel intra-planta sería más eficiente como emisor de señales acerca del estado y severidad del ataque. El daño punteado funcionaría como señal confiable de riesgo y la selección natural favorecería aquellas plantas que apuesten a una rápida inducción para la reasignación a la producción de frutos (Karban, Agrawal, Thaler, \& Adler, 1999; Schultz, Appel, Ferrieri, \& Arnold, 2013).

En $V$. breviflora una gran porción de la variación en pafr $(76.84 \%)$ fue explicada a nivel intra-planta. La variación intra-individual tiene implicaciones en la adecuación ya que es percibida por antagonistas y mutualistas, contribuye al mantenimiento de las estrategias de resistencia en las plantas y relaja las presiones selectivas que promueven la evolución de la resistencia de los herbívoros (Winn, 1996; Brennan \& Weinbaum, 2001; Brennan, Weinbaum, Rosenheim, \& Karban, 2001; Sobral, Guitián, Guitián, \& Larrinaga, 2013). La variación subindividual podría ser explicada por numerosos factores, tales como distribución diferencial de luz, agua, nutrientes, capacidad fotosintética y metabolitos de defensas (mosaico fotoquímico) (Powell \& Raffa, 1999; Wilson \& Faeth, 2001; Honěk \& Martinková, 2002; Meyer, Roces, $\&$ Wirth, 2006). Consecuentemente, los altos valores de variación en el daño podrían interpretarse como diferencias en la percepción de la variación por parte de los distintos grupos funcionales de herbívoros.

La relación entre la variación en los rasgos foliares morfológicos área foliar (af), relación longitud/ancho (laf) y fenológicos (edad de la hoja) con la magnitud del daño foliar (pafr y afr) muestran que la pafr se incrementó a mayor laf y el afr se incrementó a mayor af y mayor edad de la hoja. Esto es, hojas de mayor longitud fueron mas consumidas por herbívoros y hojas que aumentaron su tamaño con la edad también exhibieron mayor proporción de área foliar removida acumulada. Estudios previos señalan que las características foliares morfológicas, como apariencia visual, dureza, cera y presencia/ausencia de tricomas (Feeny, 1976; Edwards, 1982; Prokopy \& Owens, 1983; Kovalev, Filippov, \& Gorb, 2013) son factores influyentes en la elección y eficiencia en el uso del recurso por parte de los insectos, por tal razón el tamaño y la apariencia foliar, que viene determinada por la edad de la hoja, condicionarían los niveles de daño.

Específicamente el impacto de la forma de la hoja en el comportamiento de alimentación y/u oviposición ha sido menos estudiado, aún con el conocimiento (mayormente en minadores) sobre su importancia cómo factor limitante de la tasa de alimentación y acceso a ciertas 
partes de la hoja por parte de los herbívoros (Brown et al., 1991; Royer, 2012). Cárdenas et al. (2014) exploraron el papel modulador de niveles de herbivoría sobre la variación en rasgos fisiológicos, químicos y morfológicos, a nivel inter e intra-específico. Ellos detectaron alta variabilidad en la magnitud del daño a los dos niveles, y sugirieron que el área foliar sería un buen predictor de los niveles de herbivoría (Kurokawa \& Nakashizuka, 2008; Schuldt et al., 2012). Por otro lado, la edad de la hoja es un rasgo que guarda relación con la atracción a los herbívoros y se ha observado que las hojas jóvenes tienden a exhibir mas daño que las hojas maduras (Marquis, 1991; Kursar \& Coley, 2003; Carmona, Lajeunesse, \& Johnson, 2011; Agrawal \& Weber, 2015). Sin embargo, nuestros resultados mostraron que las hojas viejas presentan mayor pafr que las nuevas, lo que respondería a un mayor tiempo de exhibición y de disponibilidad para los herbívoros (Fenny, 1976). Adicionalmente, concordaría con la teoría de la defensa óptima, donde los órganos más valiosos, en término de aporte al éxito reproductivo (en este caso las hojas nuevas), son mejor defendidos que otros órganos menos valiosos (hojas viejas) dentro de una planta (Rhoades, 1979, Badenes-Perez, Reichelt, Gershenzon, \& Heckel, 2014).

La diferencia en el incremento de la pafr entre La Horqueta y V. P. Monti con respecto a los demás sitios (A. Chiquitas y San Ignacio), se relacionaría con los diferentes patrones de forrajeo de los grupos funcionales de herbívoros. La variación en los patrones de forrajeo sería el resultado de factores físicos externos, como distribución espacial de las plantas, temperatura y humedad o factores biológicos, como identidad del insecto, tamaño del cuerpo, tipo de aparato bucal o aspectos comportamentales ligados a las estrategias de evasión de depredadores (Heinrich, 1979; Dirzo, 1984; Schowalter, Hargrove \&, Crossley, 1986; Morrison \& Reekie, 1995; Li, Luo, Tian, Peng, \& Zhou, 2012). El daño cortador produjo mayores valores de pafr y tuvo mayor frecuencia de ocurrencia en los sitios La Horqueta y V. P. Monti, lo que sugiere que las especies de insectos con aparato bucal de tipo masticador son los herbívoros más abundantes en estos sistemas. El daño punteado es el tipo de daño mas frecuente en $V$. breviflora (Valoy, sin publicar). El análisis reveló una relación negativa entre el daño punteado y la pafr, lo que sugiere una ventaja para la planta, en términos de pérdida de tejido foliar. Si se asume que el tipo de daño es la impronta visible e indirecta de las características foliares (morfológicas, químicas, nutricionales o fotosintéticas) que son blanco de la selección mediada por herbívoros, se esperaría que la selección natural favorezca ciertas distribuciones y combinaciones de rasgos foliares que se manifiesten en un patrón de daño punteado.

En definitiva, el análisis de selección reforzaría la idea acerca de que la selección natural favorece el daño punteado con respecto a los cortadores y minadores, dado que prevalecería como una estrategia de ahorro de recursos (Mauricio et al., 1993, Avila-Sakar, Leist, \& Stephenson, 2003; Li et al., 2012) o para minimizar costos de movilización y transporte de asimilados.

En conjunto, se detectó que los herbívoros moldean el fenotipo de las plantas a través de diversas percepciones de los rasgos y generan patrones selectivos similares con diferente magnitud según el grupo funcional de herbívoros. La variación en rasgos foliares formaría parte de las estrategias adaptativas de las plantas. Este trabajo muestra la importancia de cómo los rasgos foliares, grupos funcionales de herbívoros y los patrones de defensa de las plantas constituyen una matriz inicial selectiva que modularía las características del despliegue de flores y frutos y en consecuencia la relación de las plantas con los socios mutualistas.

Declaración de ética: los autores declaran que todos están de acuerdo con esta publicación y que han hecho aportes que justifican su autoría; que no hay conflicto de interés de cualquier tipo; y que han cumplido con todos los requisitos y procedimientos éticos y legales pertinentes. El documento firmado se encuentra en los archivos de la revista. 


\section{AGRADECIMIENTOS}

A Martín Portal, a Plásticos La Rioja y a Hugo Ayarde por sus valiosos aportes al manuscrito. Este estudio fue financiado parcialmente por la Fundación Miguel Lillo, el Ministerio de Educación y el Consejo Nacional de Investigaciones Científicas y Técnicas (CONICET) de la República Argentina (PIP 11420110100395 otorgado a M.O.).

\section{RESUMEN}

La selección mediada por herbívoros moldea la evolución de los caracteres defensivos en las plantas. El conocimiento acerca del rol de los herbívoros como mediadores de selección es escaso y más aún si se consideran los grupos funcionales de herbívoros. Los objetivos de este trabajo fueron (1) describir la variación en rasgos foliares entre poblaciones, entre plantas dentro de una población y a nivel sub-individual (intra-planta), (2) explorar la relación entre la variación en el nivel de herbivoría y rasgos foliares, (3) determinar la relación entre rasgos foliares y patrones de daño y (4) estimar los regímenes de selección por diferentes grupos funcionales de herbívoros. Realizamos este estudio en cuatro poblaciones de Vassobia breviflora en el noroeste de Argentina (Yungas). Los rasgos foliares considerados fueron: tamaño, área foliar (af), forma (relación longitud/ancho de la hoja; laf) y proporción de área foliar removida (pafr) ( $\mathrm{N}=1582$ hojas, 57 plantas). Los herbívoros consumieron $15.6 \%$ del área foliar y $76.8 \%$ de la variación en la pafr ocurrió a nivel sub-individual. El patrón de daño fue dominado por herbívoros cortadores (70 $\%$ ), seguido de un patrón de herbivoría punteada (22\%), 5 $\%$ mixto y $1 \%$ minador. Se detectó selección no lineal para laf $(\gamma \mathrm{ii}=-0.180, \mathrm{EE}=0.76, \mathrm{P}<0.05)$, y selección correlacional entre el daño cortador y af $(\gamma \mathrm{ij}=-1.297, \mathrm{SE}=0.62, \mathrm{P}$ $<0.05)$ y entre el daño punteado y af $(\gamma \mathrm{ij}=-1.130, \mathrm{SE}=0.76$, $\mathrm{P}<0.05)$. La selección natural favoreció plantas con hojas pequeñas y alta remoción foliar y hojas grandes con menor daño y se detectó selección en contra de hojas grandes con mayor daño. Además, deducido de la relación entre el tipo de daño y la adecuación relativa, la selección favorecería el daño punteado por sobre el cortador. Las plantas resolverían el conflicto con los herbívoros según el tipo de daño y la selección natural regularía el despliegue foliar como una estrategia para lidiar con la diversidad de herbívoros.

Palabras clave: interacciones planta-animal; selección fenotípica; grupos funcionales; rasgos foliares; niveles de herbivoría; patrones de daño.

\section{REFERENCIAS}

Agrawal, A. A., \& Weber, M. G. (2015). On the study of plant defence and herbivory using comparative approaches: how important are secondary plant compounds. Ecology Letters, 18, 985-991.

Anton, A. M., \& Zuloaga, F. O. (2013). Recuperado de http://www.floraargentina.edu.ar

Ågren, J., Hellström, F., Toräng, P., \& Ehrlén, J. (2013). Mutualists and antagonists drive among-population variation in selection and evolution of floral display in a perennial herb. Proceedings of the National Academy of Sciences, 110, 18202-18207.

Avila- Sakar, G., Leist, L. L., \& Stephenson, A. G. (2003). Effects of the spatial pattern of leaf damage on growth and reproduction: nodes and branches. Journal of Ecology, 91, 867-879.

Ayarde, H. (2005). Hidrología en bosques subtropicales de montaña en Argentina. En M. Ataroff, \& J. Silva (Eds.), Dinámica Hídrica en Sistemas Neotropicales (pp. 37-42). Mérida, Venezuela: ICAE, Universidad de Los Andes.

Badenes-Perez, F. R., Reichelt, M., Gershenzon, J., \& Heckel, D. G. (2014). Using plant chemistry and insect preference to study the potential of Barbarea (Brassicaceae) as a dead-end trap crop for diamondback moth (Lepidoptera: Plutellidae). Phytochemistry, 98, 137-144.

Baldwin, I. T., \& Schultz, J. C. (1983). Rapid changes in tree leaf chemistry induced by damage: evidence for communication between plants. Science, 221, 277-279.

Bernacki F. G. (2014). Biología floral y frutal de Vassobia breviflora (Sedtn.) Hunz. (Solanaceae) en el noroeste argentino (Tesis de licenciatura). Universidad Nacional de Tucumán, Argentina.

Bernacki, F. G., Albornoz, P., Valoy, M., \& Ordano, M. (2015). Anatomía de flor y fruto de Vassobia breviflora (Solanaceae) en el sur de las Yungas Australes (Argentina). Phyton-International Journal of Experimental Botany, 82, 478-487.

Bernays, E., \& Graham, M. (1988). On the evolution of host specificity in phytophagous arthropods. Ecology, $69,886-892$.

Bianchi, A. R., \& Yáñez, C. E. (1992). Las Precipitaciones en el Noroeste Argentino. Salta, Argentina: Ediciones Guadarrama Madrid.

Brennan, E. B., \& Weinbaum, S. A. (2001). Performance of adult psyllids in nochoice experiments on juvenile 
and adult leaves of Eucalyptus globulus. Entomologia Experimentalis et Applicata, 100, 179-185.

Brennan, E. B., Weinbaum, S. A., Rosenheim, J. A., \& Karban, R. (2001). Heteroblasty in Eucalyptus globulus (Myricales:Myricaceae) affects ovipositional and settling preferences of Ctenarytaina eucalypti and C. spatulata (Homoptera:Psyllidae). Environmental Entomology, 30, 1144-1149.

Breheny, P., \& Burchett, W. (2015). Visualization of regression models using visreg. Recuperado de https:// cran.r-project.org/web/packages/visreg/index

Brown, V. K., Lawton, J. H., \& Grubb, P. J. (1991). Herbivory and the evolution of leaf size and shape [and discussion]. Philosophical Transactions of the Royal Society of London. Series B: Biological Sciences, 333(1267), 265-272.

Brown, A. D., \& Corcuera, J. (1999). Donación de nuevas áreas protegidas en las Yungas de la Argentina (Informe Técnico). Argentina: LIEY/FVSA.

Brown, A. D., Grau, A., Lomáscolo, T., \& Gasparri, N. I. (2002). Una estrategia de conservación para las selvas subtropicales de montaña (Yungas) de Argentina. Ecotropicos, 15, 147-159.

Cabrera, A. L. (1976). Territorios fitogeográficos de la Republica Argentinas. En W. F. Kugler (Eds.), Enciclopedia Argentina de Agricultura y Jardinería, 2, 1-85.

Cabrera, A. L. (1979). Solanaceae. En A. Burkart (Eds.), Flora Ilustrada de Entre Ríos V (Argentina) (pp. 346452). Buenos Aires, Argentina: Instituto Nacional de Tecnología Agropecuaria.

Campitelli, B. E., Simonsen, A. K., Wolf, A. R., Manson, J. S., \& Stinchcombe, J. R. (2008). Leaf shape variation and herbivore consumption and performance: a case study with Ipomoea hederacea and three generalists. Arthropod-Plant Interactions, 2, 9-19.

Canto Aguilar, M. A. (2004). Herbivoría crecimiento y reproducción de Anthurium schlechtendalii Kunth (Araceae) en ecosistemas diferentes (Tesis doctoral). Xalapa, Veracruz, México.

Cárdenas, R. E., Valencia, R., Kraft, N. J., Argoti, A., \& Dangles, O. (2014). Plant traits predict inter-and intraspecific variation in susceptibility to herbivory in a hyperdiverse Neotropical rain forest tree community. Journal of Ecology, 102, 939-952.

Carmona, D., Lajeunesse, M. J., \& Johnson, M. T. J. (2011). Plant traits that predict resistance to herbivores. Functional Ecology, 25, 358-367.

Chen, Y. S., Chesson, P., Wu, H. W., Pao, S. H., Liu, J. W., Chien, L. F., ... Sheue, C. R. (2017). Leaf structure affects a plant's appearance: combined multiple-mechanisms intensify remarkable foliar variegation. Journal of Plant Research, 130, 311-325.

Coley, P. D., Bryant, J. P., \& Chapin, F. S. (1985). Resource Availability and Plant Antiherbivore Defense. Science, 230, 895-899.

Crawley, M. J. (1997). Plant-herbivore dynamics. In M. J. Crawley (Eds.), Plant Ecology (pp. 401-474). Oxford, England: Blackwell Science.

Crawley, M. J. (2007). The R book. West Sussex, England: John Wiley \& Sons.

Digilio, A. P., \& Legname, P. R. (1966). Los árboles indígenas de la Provincia de Tucumán. Opera Lilloana, $15,1-107$.

Dirzo, R. (1984). Herbivory: a phytocentric overview. In R. Dirzo, \& J. Sarukhán (Eds.), Perspectives on plant population ecology (pp. 141-165). Sunderland, USA: Sinauer Associates Inc.

Edwards, P. B. (1982). Do waxes on Eucalyptus leaves provide protection from grazing insects? Australian Journal of Ecology, 7, 347-352.

Feeny, P. (1976). Plant apparency and chemical defense. In J. Wallace, \& R. L. Mansell (Eds.), Biochemical interaction between plants and insects. Recent advances in phytochemistry (pp. 1-40). USA: Springer.

Ferrari, S. L. P., \& Cribari-Ñeto, F. (2004). Beta Regression for Modeling Rates and Proportions. Journal of Applied Statistics, 31, 799-815.

Geber, M. A., \& Griffin, L. A. (2003). Inheritance and natural selection on functional traits. International Journal of Plant Sciences, 164, S21-S42.

Gelman, A., \& Hill, J. (2007). Data analysis using regression and multilevel/ hierarchical models. Cambridge, UK: Cambridge University Press.

Gerasymenko, V. (2017). The Model of Fitness in a Heterogeneous Environment on Reaction Norms. Proceedings of the Latvian Academy of Sciences, 71(4), 303-306.

Giannini, N. P. (1999). La interacción de aves-murciélagos-plantas en el sistema de frugivoría y dispersión de las semillas en San Javier, Tucumán, Argentina (Tesis doctoral). Universidad Nacional de Tucumán, Tucumán, Argentina.

Gilbert, L. E. (1979). Development of theory in the analysis of insect-plant interactions. Analysis of Ecological Systems, 117-154.

Givnish, T. J. (1990). Leaf mottling: relation to growth form and leaf phenology and possible role as camouflage. Functional Ecology, 463-474. 
Hare, D. (2012). How insect herbivores drive the evolution of plants. Science, 338, 50-51.

Heinrich, B. (1979). Foraging strategies of caterpillars. Oecologia, 42, 325-337.

Hendrix, S. D. (1988). Herbivory and its impact on plant reproduction. Plant Reproductive Ecology, 246-263.

Herrera, C. M. (2009). Multiplicity in unity: plant subindividual variation and interaction with animals. Chicago, USA: University of Chicago Press.

Hikosaka, K. (2005). Leaf canopy as a dynamic system: ecophysiology and optimality in leaf turnover. Annals of Botany, 95, 521-533.

Honêk, A., \& Martinková, Z. (2002). Factors of between- and within- plant distribution of Metopolophium dirhodum (Homoptera: Aphididae) on small grain cereals. Journal of Applied Entomology, 126, 378-383.

Hueck, K. (1978). Los bosques de Sudamérica. Ecología composición e importancia económica. Eschborn, República Federal de Alemania: GTZ.

Johnson, S. N., Clark, K. E., Hartley, S. E., Jones, T. H., McKenzie, S. W., \& Koricheva, J. (2012). Aboveground-belowground herbivore interactions: a metaanalysis. Ecology, 93, 2208-2215.

Karban, R., Agrawal, A. A., Thaler, J. S., \& Adler, L. S. (1999). Induced plant responses and information content about risk of herbivory. Trends in Ecology \& Evolution, 14, 443-447.

Kovalev, A. E., Filippov, A. E., \& Gorb, S. N. (2013) Insect wet steps: loss of fluid from insect feet adhering to a substrate. Journal of the Royal Society Interface, 10, 201-239.

Kurokawa, H., \& Nakashizuka, T. (2008). Leaf herbivory and decomposability in a Malaysian tropical rain forest. Ecology, 89, 2645-2656.

Kursar, T. A., \& Coley, P. D. (2003). Convergence in defense syndromes of young leaves in tropical rainforests. Biochemical Systematics and Ecology, 31, 929-949.

Lande, R., \& Arnold, S. J. (1983). The measurement of selection on correlated characters. Evolution, $1210-1226$

Lehndal, L. \& Ågren, J. (2015). Latitudinal variation in resistance and tolerance to herbivory in the perennial herb Lythrum salicaria is related to intensity of herbivory and plant phenology. Journal of Evolutionary Biology, 28, 576-589.

Lehtilä, K. (1999). Impact of herbivore tolerance and resistance on plant life histories. In T. Vuorisalo, \& P. Mutikainen (Eds.), Life-history Evolution in Plants (pp. 303-328). Dordrecht, Netherlands: Kluwer Academic Publishers.

Lev-Yadun, S. (2016). Plants are not sitting ducks waiting for herbivores to eat them. Plant Signaling \& Behavior, 11(5), e1179419.

Li, W., Luo, J., Tian, X., Peng, C., \& Zhou, X. (2012). Patterns of defoliation and their effect on the plant growth and photosynthetic characteristics of Ipomoea cairica. Weed Biology and Management, 12, 40-46.

Linhart, Y. B., \& Grant, M. C. (1996). Evolutionary significance of local genetic differentiation in plants. Annual Review of Ecology and Systematics, 27, 237-277.

Marquis, R. J. (1991). Herbivore fauna of Piper (Piperaceae) in a Costa Rican wet forest: Diversity, specificity and impact. In P. W. Price, T. M. Lewinsohn, G. W. Fernandes, \& W. W. Benson (Eds.), Plant-Animal Interactions: Evolutionary Ecology in Tropical and Temperate Regions (pp. 197-208). New York, USA: Wiley \& Sons.

Marquis, R. J. (1992). The selective impact of herbivores. In R. S Fritz, \& E. L Simms (Eds.), Plant resistance to herbivores and pathogens (pp. 301-325). Chicago, USA: University of Chicago Press.

Marquis, R. J., Salazar, D., Baer, C., Reinhardt, J., Priest, G., \& Barnett, K. (2016). Ode to Ehrlich and Raven or how herbivorous insects might drive plant speciation. Ecology, 97, 2939-2951.

Mauricio, R., Bowers, M. D., \& Bazzaz, F. A. (1993). Pattern of leaf damage affects fitness of the annual plant Raphanus sativus (Brassicaceae). Ecology, 2066-2071.

MacColl, A. D. (2011). The ecological causes of evolution. Trends in Ecology \& Evolution, 26, 514-522.

Meyer, S. T., Roces, F., \& Wirth, R. (2006). Selecting the drought stressed: effects of plant stress on intraspecific and within- plant herbivory patterns of the leafcutting ant Atta colombica. Functional Ecology, 20, 973-981.

Morrison, K. D., \& Reekie, E. G. (1995). Pattern of defoliation and its effect on photosynthetic capacity in Oenothera biennis. Journal of Ecology, 83, 759-767.

Nguyen, D., Rieu, I., Mariani, C., \& van Dam, N. M. (2016). How plants handle multiple stresses: hormonal interactions underlying responses to abiotic stress and insect herbivory. Plant Molecular Biology, 91(6), 727-740.

O’Neill, G. A., Aitken, S. N., King, J. N., \& Alfaro, R. I. (2002). Geographic variation in resin canal defenses in seedlings from the Sitka spruce $\mathrm{x}$ white spruce introgression zone. Canadian Journal of Forest 
Research-Revue Canadienne De Recherche Forestiere, 32, 390-400.

Palacio, F. X., Valoy, M., Bernacki, F., Sánchez, M. S., Núñez-Montellano, M. G., Varela, O., \& Ordano, M. (2017). Bird fruit consumption results from the interaction between fruit-handling behaviour and fruit crop size. Ethology Ecology \& Evolution, 29, 24-37.

Pigliucci, M. (2003). Phenotypic integration: studying the ecology and evolution of complex phenotypes. Ecology Letters, 6, 265-272.

Pilson, D. (2000). The evolution of plant response to herbivory: simultaneously considering resistance and tolerance in Brassica rapa. Evolutionary Ecology, 14, 457-489.

Pinehiro, J., Bates, D., DebRoy, S., \& Sarkar, D. (2012) NLME: Linear and nonlinear mixed effects models. Recuperado de http://cran.r-project.org/web/packages/nlme/nlme.pdf

Powell, J. S., \& Raffa, K. F. (1999). Sources of variation in concentration and composition of foliar monoterpenes in tamarack (Larix laricina) seedlings: roles of nutrient availability, time of season, and plant architecture. Journal of Chemical Ecology, 25, 1771-1797.

Propoky, R. J., \& Owens, E. D. (1983). Visual detection of plants by herbivorous insects. Annual Review of Entomology, 28, 337-364.

R Core Team (2013). R: A language and environment for statistical computing. Vienna, Austria: R Foundation for Statistical Computing. Recuperado de http:// www.R-project.org/

Rasband, W. (1997). ImageJ. National Institutes of Health, USA. Recuperado de http://rsb.info.nih.gov/ij

Rhoades, D. F. (1979). Evolution of plant chemical defense against herbivores. In G. A Rosenthal, \& D. H Janzen (Eds.), Herbivores: Their Interactions with Secondary Plant Metabolites (pp. 3-54). New York, USA: Academic Press.

Royer, D. L. (2012). Leaf Shape Responds to Temperature but not CO 2 in Acer rubrum. PloS one, 7, e49559.

Santillán de Andrés, S., Barbieri de Santamarina, E., Ricci, T. R., \& Würschmidt, E. J. (1967). La región de las Sierras del Nordeste de la provincia de Tucumán. Tucumán: Universidad Nacional de Tucumán, Departamento de Geografía, Facultad de Filosofía y Letras. Serie Monográfica, 16, 1-89.

Sarkar, D. (2015). Lattice: Multivariate Data Visualization with $R$. New York, USA: Springer.

Schmitt, T. M., Hay, M. E., \& Lindquist, N. (1995). Constraints on chemically mediated coevolution: multiple functions for seaweed secondary metabolites. Ecology, 107-123.
Schowalter, T. D., Hargrove, W., \& Crossley, D. A. (1986). Herbivory in forested ecosystems. Annual Review of Entomology, 31, 177-196.

Schuldt, A., Bruelheide, H., Durka, W., Eichenberg, D., Fischer, M., \& Kröber, W. (2012). Plant traits affecting herbivory on tree recruits in highly diverse subtropical forests. Ecology Letters, 15, 732-739.

Schultz, J. C., Appel, H. M., Ferrieri, A. P., \& Arnold, T. M. (2013). Flexible resource allocation during plant defense responses. Frontiers in Plant Science, 4.

Shimada, T., Takahashi, A., Shibata, M., \& Yagihashi, T. (2015). Effects of within plant variability in seed weight and tannin content on foraging behaviour of seed consumers. Functional Ecology, 29, 1513-1521.

Simas, A. B., \& Rocha, A. V. (2015). betareg: Beta Regression. R package version 3.0-5, URL. Recuperado de http://r-project.org/src/contrib/Archive/betareg

Sobral, M., Guitián, J., Guitián, P., \& Larrinaga, A. R. (2013). Selective pressure along a latitudinal gradient affects subindividual variation in plants. PloS one, $8, \mathrm{e} 74356$.

Strauss, S. Y., Conner, J. K., \& Lehtilä, K. P. (2001). Effects of foliar herbivory by insects on the fitness of Raphanus raphanistrum: damage can increase male fitness. The American Naturalist, 158, 496-504.

Strauss, S. Y., Rudgers, J. A., Lau, J. A., \& Irwin, R. E. (2002). Direct and ecological costs of resistance to herbivory. Trends in Ecology \& Evolution, 17, 278-285.

Taura, H. M., \& Laroca, S. (2004). Biologia da polinização: interações entre as abelhas (Hym., Apoidea) e as flores de Vassobia breviflora (Solanaceae) [Pollination biology: interactions between bees and flowers of Vassobia breviflora (Solanaceae)]. Acta Biologica Paranaense, 33, 143-162.

Thompson, J. N. (2005). The Geographic Mosaic of Coevolution. Chicago, USA: University of Chicago Press.

Valoy, M., Ordano, M., \& Benavídez, A. (2012). Herbivoría foliar y autonomía de ramas en Psychotria carthagenensis (Rubiaceae). Lilloa, 49, 68-77.

Valverde, P. L., Fornoni, J., \& Núñez-Farfán, J. (2001). Defensive role of leaf trichomes in resistance to herbivorous insects in Datura stramonium. Journal of Evolutionary Biology, 14, 424-432.

Warner, P. J., \& Cushman, H. J. (2002). Influence of herbivores on a perennial plant: variation with life history stage and herbivore species. Oecologia, 132, 77-85.

Wade, M. J., \& Kalisz, S. (1990). The causes of Natural Selection. Evolution, 44, 1947-1955. 
Weis, A. E., \& Gorman, W. L. (1990). Measuring selection on reaction norms: an exploration of the Eurosta Solidago system. Evolution, 44, 820-831.

Weis, A. E., Abrahamson, W. G., \& Andersen, M. C. (1992). Variable selection on Eurosta's gall size, I: the extent and nature of variation in phenotypic selection. Evolution, 46, 1674-1697.

Whitham, T. G. (1983). Host manipulation of parasites: within-plant variation as a defense against rapidly evolving pests. In R. F. Denno, \& M. S. McClure (Eds.), Variable Plants and Herbivores in Natural and Managed Systems (pp. 15-41). New York, USA: Academic Press.
Wilson, D., \& Faeth, S. H. (2001). Do fungal endophytes result in selection for leafminer ovipositional preference? Ecology, 82, 1097-1111.

Winn, A. A. (1996). The contributions of programmed developmental change and phenotypic plasticity to within-individual variation in leaf traits in Dicerandra linearifolia. Journal of Evolutinary Biology, 9, 737-752.

Zuur, A. F., Ieno, E. N., Walker, N. J., Saveliev, A. A., \& Smith, G. M. (Eds.) (2009). In Mixed effects modelling for nested data. Mixed effects models and extensions in ecology with $R$ (pp. 101-142). New York, USA: Springer.

\section{APÉNDICE 1}

Estadística descriptiva para variables de herbivoría en cuatro poblaciones de $V$. breviflora Descriptive statistics for herbivory variables in four $V$. breviflora populations

\begin{tabular}{|c|c|c|c|c|c|}
\hline \multicolumn{6}{|c|}{ Aguas chiquitas } \\
\hline & media & mínimo & máximo & sd & $\mathrm{cv}$ \\
\hline pafr & 0.085 & 0.0005 & 0.736 & 0.118 & 1.386 \\
\hline afr & 2.781 & 0.015 & 35.330 & 3.965 & 1.425 \\
\hline af & 20.878 & 3.500 & 89.570 & 11.418 & 0.546 \\
\hline laf & 1.910 & 0.860 & 9.140 & 0.489 & 0.256 \\
\hline \multicolumn{6}{|c|}{ La Horqueta } \\
\hline pafr & 0.144 & 0.011 & 0.762 & 0.165 & 1.146 \\
\hline afr & 2.122 & 0.097 & 9.733 & 1.958 & 0.920 \\
\hline af & 9.647 & 2.168 & 31.732 & 5.514 & 0.572 \\
\hline laf & 2.128 & 1.215 & 3.791 & 0.52 & 0.244 \\
\hline \multicolumn{6}{|c|}{ V.P. Monti } \\
\hline pafr & 0.199 & 0.003 & 0.664 & 0.14 & 0.707 \\
\hline afr & 3.622 & 0.026 & 30.701 & 4.07 & 1.124 \\
\hline af & 16.896 & 3.279 & 54.525 & 9.748 & 0.576 \\
\hline laf & 2.523 & 1.109 & 23.382 & 1.634 & 0.647 \\
\hline \multicolumn{6}{|c|}{ San Ignacio } \\
\hline pafr & 0.119 & 0.003 & 0.441 & 0.112 & 0.943 \\
\hline afr & 1.741 & 0.007 & 9.412 & 1.674 & 0.961 \\
\hline af & 10.370 & 0.998 & 34.689 & 6.847 & 0.660 \\
\hline laf & 2.060 & 1.305 & 4.851 & 0.500 & 0.242 \\
\hline
\end{tabular}

Pafr = Proporción de área foliar removida, $\mathbf{a f r}=$ Área foliar removida, laf = Relación longitud/ancho foliar. $\mathbf{P a f r}=$ Proportion of leaf area removed, $\mathbf{a f r}=$ Leaf area removed, laf $=$ leaf length $/$ width ratio. 